\title{
New approach to estimating variability in visual field data using an image processing technique
}

School of

Mathematics,

Actuarial Science and

Statistics, City

University, London

D P Crabb

H P Wynn

Applied Vision

Research Centre, City

University, London

D F Edgar

Department of Visual Science, Institute of

Ophthalmology,

London

F W Fitzke

Glaucoma Unit, Moorfields Eye Hospital, London A I McNaught

Correspondence to: F W Fitzke, Department of Visual Science, Institute of Ophthalmology, 11 Bath Street, London EC1V 9EL Accepted for publication 20 October 1994

\author{
David P Crabb, David F Edgar, Frederick W Fitzke, Andrew I McNaught, Henry P Wynn
}

\begin{abstract}
Aims-A new framework for evaluating pointwise sensitivity variation in computerised visual field data is demonstrated.

Methods-A measure of local spatial variability (LSV) is generated using an image processing technique. Fifty five eyes from a sample of normal and glaucomatous subjects, examined on the Humphrey field analyser (HFA), were used to illustrate the method.

Results-Significant correlation between LSV and conventional estimates - namely, HFA pattern standard deviation and short term fluctuation, were found.

Conclusion-LSV is not dependent on normals' reference data or repeated threshold determinations, thus potentially reducing test time. Also, the illustrated pointwise maps of LSV could provide a method for identifying areas of fluctuation commonly found in early glaucomatous field loss.
\end{abstract}

(Brf Ophthalmol 1995; 79: 213-217)

Automated perimetry is widely used for the detection and follow up of glaucomatous field loss. Evaluation of the pointwise sensitivity or threshold variation in visual field data constitutes an important component of perimetric assessment. Indeed, the behaviour of early glaucomatous field loss is characterised by this variability and fluctuation. ${ }^{1-4}$

The Humphrey field analyser (HFA) is typical of advanced perimeters in providing the clinician with a battery of devices to quantify and interpret this pointwise sensitivity variation and threshold fluctuation. STATPAC ${ }^{5}$ (HFA's results analysis package) produces pattern deviation maps of the field by comparing the tested thresholds at each location to age corrected normal values adjusted to the subject's hill of vision. Locations that have a low probability of occurring in the normal database are flagged. The extent of all the deviations is summarised by the pattern standard deviation (PSD) index. Short term fluctuation (SF) is partly a measure of subject response variability. STATPAC calculates SF during a field examination as a weighted mean of the differences at 10 preselected locations where the threshold value is determined twice.

SF, pattern deviations maps, PSD, and CPSD (pattern standard deviations corrected by SF) are widely used by clinicians in their diagnosis and management of ocular diseases. However, the actual values adopted for the age corrected normal reference data are subject to debate. ${ }^{6-8}$ Furthermore, a test duration fatigue effect has been widely reported in normal and glaucomatous subjects. ${ }^{9-11}$ These research findings question the suitability of the normal reference field technique for evaluating pattern deviation maps, PSD and, with regards to fatigue factors, the time consuming double threshold determination strategy for estimating SF.

This paper illustrates a new framework for estimating pointwise sensitivity variation and fluctuation. The rationale exploits the inherent spatial dependence which exists between neighbouring sensitivity values in the field ${ }^{12}$ and adheres to the trend of developing procedures that take this important relation into consideration. ${ }^{1314}$ Simply, the difference between a sensitivity value and a suitable average of its neighbours is assumed to be a measure of the variance at that location. The size, direction, and site of these differences reflect the level of variability and non-uniform deviation across the field. An image processing technique was used to reduce the variability in visual field data in the previous paper. ${ }^{15}$ Here the same spatial filter process is applied, but additionally the level of extracted pointwise variability is quantified to give a new measure of local spatial variability (LSV). The method is demonstrated and simple illustrative comparisons with the conventional HFA parameters of variability are made. LSV is determined without normals' reference data or replicate threshold measurement, thus saving test time. Additionally, pointwise maps of LSV are illustrated that could provide a device for identifying areas of high variability commonly associated with glaucomatous field loss.

\section{Materials and methods}

SUBJECTS AND DATA

The sample used to illustrate these methods comprised of one visual field from each of 55 eyes. Thirty ophthalmologically normal subjects, mean age 23 (range 19-28) years had one randomly selected eye tested. These were volunteers from a student population. All had perimetric experience and mean refractive error was less than plus or minus $3 \mathrm{D}$ in each case. Twenty five fields from 25 glaucomatous eyes of patients with a mean age 63 (range 40-75) years were additionally selected. The glaucomatous eyes were selected, without prior knowledge of the image processing analysis, from a patient database. Abnormal fields from cases of high tension glaucoma (IOP $>21 \mathrm{~mm}$ $\mathrm{Hg}$ ) were selected with a wide range of defect size, depth, and spatial distribution (range of 


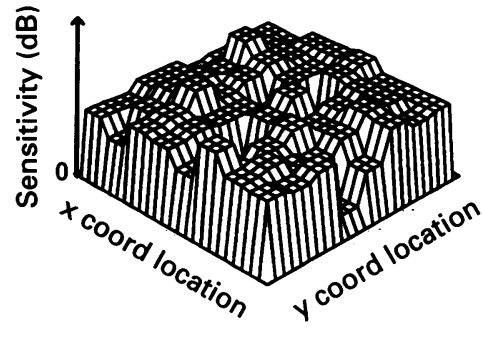

Original field ( 8 by 8 matrix)

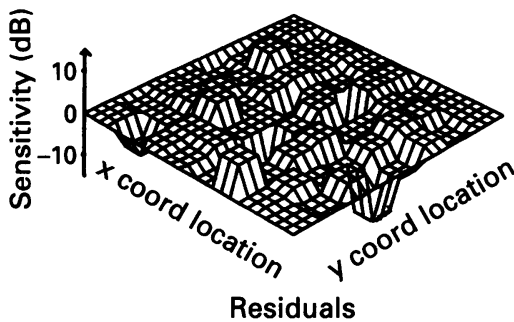

(original) - (filtered)

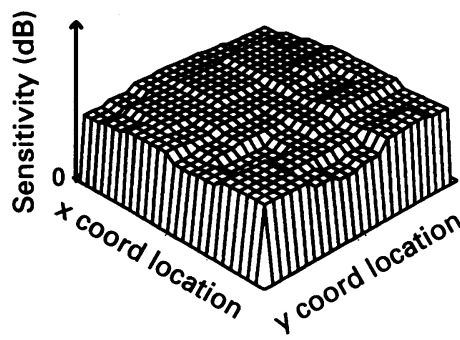

Filtered field ( 8 by 8 matrix)

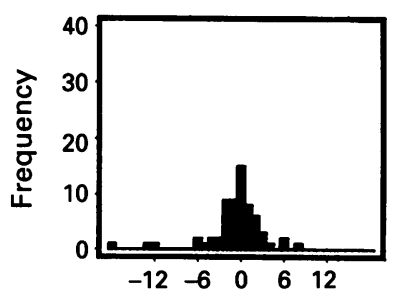

Histogram of residuals
Figure 1 A three dimensional representation of the translated 8 by 8 field with sensitivity (dB) depicted by the height of the surface. This is a field with a high level of pointwise threshold variability. Note the irregularity of the residual surface (derived from subtracting the filtered field from the original) and the spread of large residuals shown by the histogram.

MD $-0 \cdot 26$ to $-17 \cdot 93 \mathrm{~dB}$, range of PSD $2 \cdot 17$ to $13.66 \mathrm{~dB}$ ). All had optic disc appearance consistent with a diagnosis of glaucoma. The fields were selected to provide a reasonably representative sample of common patterns of glaucomatous field loss.

All subjects were tested on the HFA 630 (30-2 program) with the size III white stimulus in standard conditions. The data files comprising the results from each field examination were converted to files for a PC. The files were processed and analysed using purpose written software. The peripheral locations of the 30-2 grid (locations with $\mathrm{x}$ or $\mathrm{y}$ coordinates of +27 or -27$)$ were excluded from further analysis. Each field was thereby reduced to an 8 by 8 matrix of points with the addition of four corner locations computed using a weighted interpolation of the three adjacent thresholds.

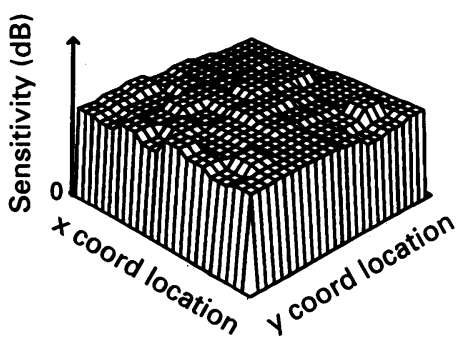

Original field ( 8 by 8 matrix)

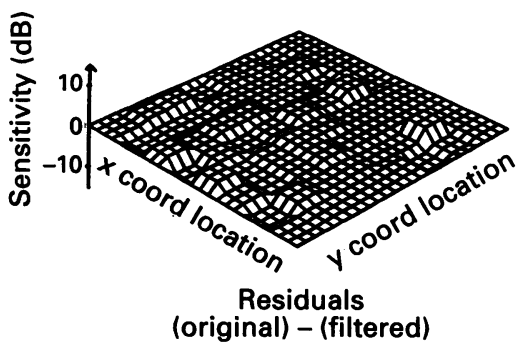

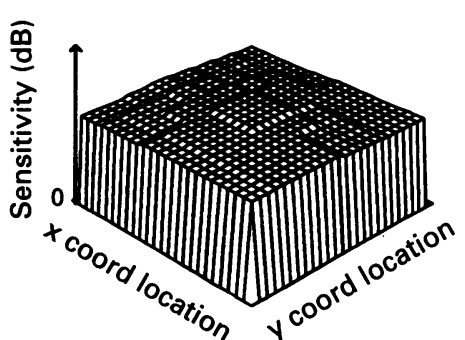

Filtered field ( 8 by 8 matrix)

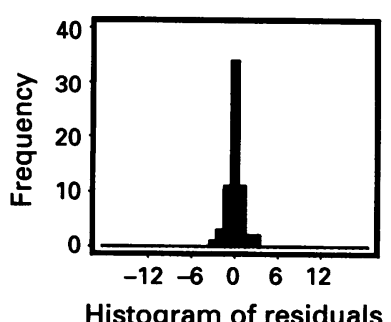

Histogram of residuals
Figure 2 A three dimensional representation of a field with a relatively low pointwise threshold variability. Note the smooth residual surface and the narrow distribution of small residuals that cluster at zero.
The two locations above and below the blind spot were excluded.

\section{IMAGE PROCESSING TECHNIQUE}

The visual field can be considered as a type of digital image composed of a matrix of light sensitivity values. In this application median filtering, described fully in the previous paper, ${ }^{15}$ is used to process sensitivity values and thus generate a smoothed version of the original field. Median filtering specifically identifies locations with extreme thresholds, and forces them to more closely resemble their neighbours. Thinking of the field as a three dimensional surface, with sensitivity $(\mathrm{dB})$ representing depth, the median filter process removes isolated spikes and troughs.

\section{LOCAL SPATIAL VARIABILITY}

A filtered version of each field was computed. The pointwise sensitivity differences (dB) between the original and filtered versions are called the residuals. It is these residuals that are assumed to describe the LSV. They are a measure of the non-uniform deviation of the field. The distribution of these residuals for each field can be expressed as a histogram. A three dimensional representation of an irregular field with a high level of pointwise threshold variability (PSD $=5 \cdot 74 \mathrm{~dB} ; \mathrm{p}<2 \%$ from STATPAC) is shown in Figure 1. In contrast Figure 2 depicts a subject with a low PSD (1.21; not significant from STATPAC).

Note particularly the difference in the size and distribution of the residuals.

Summary measures of the residuals that constitute LSV were compared with conventional HFA parameters for pointwise sensitivity variation and fluctuation. The root mean square of all the residuals was calculated for each field. Large values of this measure indicated an irregular field with many localised threshold deviations. This estimate was directly compared with PSD, as calculated by STATPAC, for all subjects. The mean of the absolute residuals from a central 6 by 6 array of the field was calculated for each subject. This area included the sites of the 10 locations where replicate threshold measurements were determined by the HFA. Comparisons between this measure of LSV and SF, as calculated by STATPAC, were also made for each subject.

\section{Results}

Scatterplots of LSV against PSD in the corresponding eyes demonstrated a clear relation in both normal and glaucomatous fields (Fig 3). Spearman's correlation coefficient (rho) is derived from a non-parametric test of ranks. It is an appropriate test of the level of association between two variables. Values greater than 0.43 are significant $(p=0.01)$ for samples of this size. There was significant correlation between LSV and PSD for normals $($ rho $=0.74)$ and glaucomatous fields $($ rho $=0.66)$. 


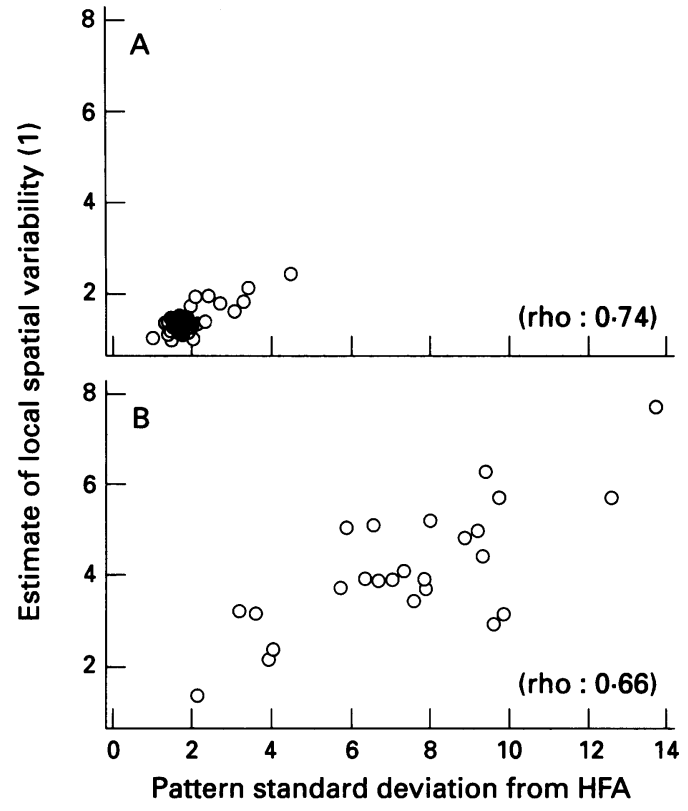

Figure 3 Scatterplot of local spatial variability (1) against pattern standard deviation (PSD) as calculated by STATPAC for (A) normals and (B) glaucomatous subjects. Each symbol represents a single field.

Significant levels of association were also observed between the second estimate of LSV and SF (normals, rho $=0.46$; patients, rho $=0.52)$. Scatterplots are shown in Figure 4. Maintaining the plots on the same scale has resulted in a clustering of the normals. Note the extreme position of one of the normal fields. This subject was singular in the normals having SF flagged as having a significantly low probability of occurring when compared with an empirical database by STATPAC $(\mathrm{p}<5 \%)$.

Figure 5 shows grey scale representations of the absolute residuals (pointwise differences between original and filtered fields) for three subjects. The location of the processed 8 by 8 matrix of values is shown relative to the HFA

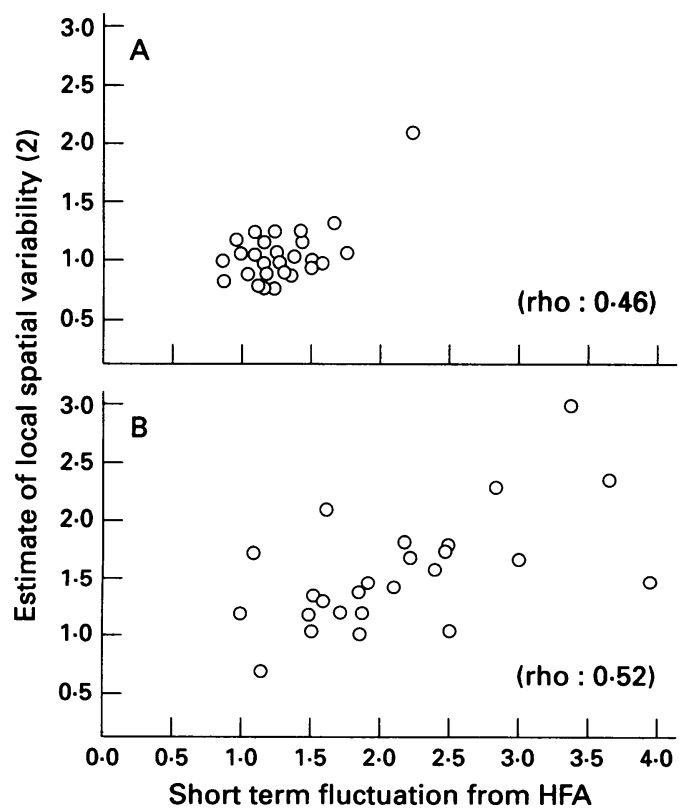

Figure 4 Scatterplot of local spatial variability (2) against short term fluctuation (SF) as calculated by STATPAC for (A) normals and (B) glaucomatous subjects.

Each symbol represents a single field.
30-2 grid outline. The small blank rectangle indicates the blind spot area (all right eyes). Dark areas depict sites of high LSV. Figures $5 A$ and $B$ are representations of the same fields shown above in Figure 1 and Figure 2, respectively. Note the difference between the field with a high level of pointwise threshold variability (a), exhibiting several areas of large residuals, compared with the normal field (b). Interestingly Figure 5C shows a glaucomatous field, with relatively low HFA parameters for variability $(\mathrm{PSD}=3.61, \quad \mathrm{p}<10 \%$; $\mathrm{CPSD}=2 \cdot 59, \quad \mathrm{p}<10 \%$ and $\mathrm{SF}=2 \cdot 12$, $\mathrm{p}<10 \%$ ). However, the grey scale map reveals the LSV is clustered and non-random in appearance which may indicate an area of further glaucomatous field decay.

\section{Discussion}

Adapted image processing techniques can potentially enhance the analysis and interpretation of visual field data. Previously such techniques were found to substantially reduce pointwise sensitivity variability on repeated testing. ${ }^{15}$ The application reported here attempts to quantify the level of variability removed by the process. The results intimate concordance between summary measures of this extracted LSV and STATPAC variables PSD and SF. It must be stressed that the value of these preliminary results is as an illustration of the methods. Future work will address the application to a large number and variety of visual fields. Other image processing techniques are being investigated to determine their effectiveness. These may prove optimum in providing a framework within which estimates of variability can be derived and cross validated with larger samples.

Local spatial variability (LSV) and the rationale that underpins its derivation clearly has advantages. Firstly, estimates of LSV are subject centred and unlike PSD or CPSD (pattern standard deviation corrected by subject's SF) are not dependent on normals' reference data. A measure of the departure from a normal height of the field for mean deviation is, however, still required. Secondly, an alternative to the current strategy for estimating SF is particularly appealing. The 10 replicate threshold measurements determined by the HFA gives the SF to an accuracy of only plus or minus $25 \% .{ }^{16}$ It has been advocated that all available double determinations should be used to improve the estimate. ${ }^{17} \mathrm{LSV}$ actually utilises information from more locations. It has been shown that replicate threshold strategies that interactively test areas close to a scotoma may prove useful. ${ }^{18}$ However, in the interest of reducing test time and subject fatigue, it is essential that such repetitions are performed only at locations that yield clinically valuable information. Significantly, LSV provides estimates of pointwise fluctuation while adopting the principle of parsimony, as no repetitions are required. This method would therefore have the advantage of reducing test time. The notion of using a single field determination to measure 
A

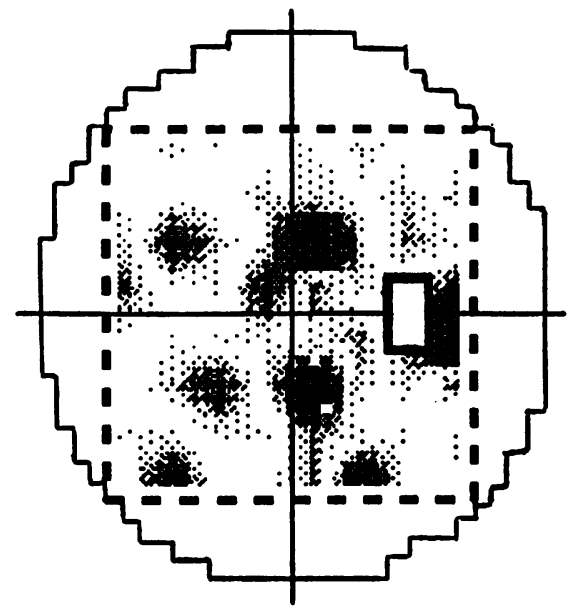

B

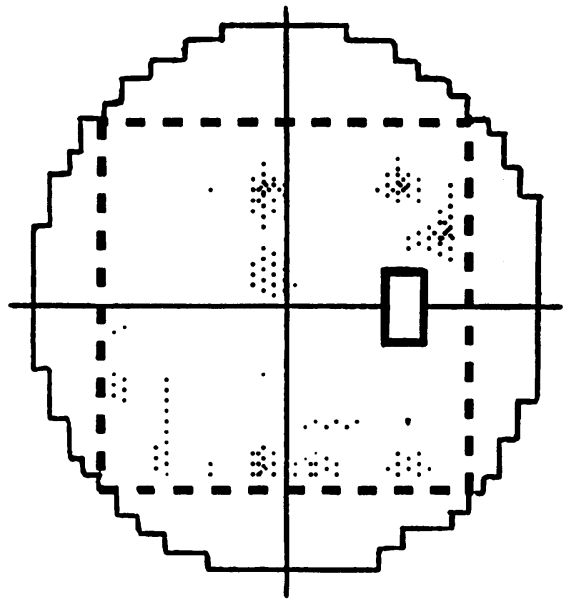

C

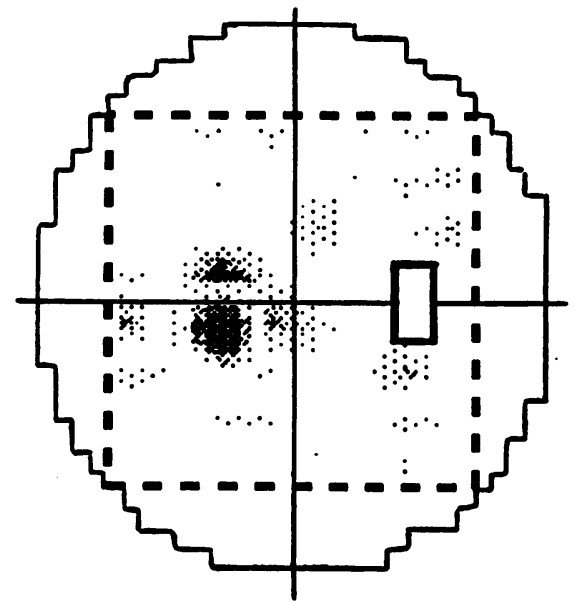

Grey scale symbols (absolute residuals)

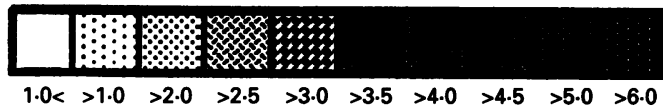

$1.0<>1.0>2.0>2.5>3.0>3.5>4.0>4.5>5.0>6.0$ Figure 5 Grey scale maps of local spatial variability for three example fields.

short term fluctuation is not new. ${ }^{19}$ However, the image processing techniques tailored to individual fields may provide a more effective framework for analysis.

A global analysis of field data does not fully describe the clinically useful information in a visual field examination. ${ }^{20} 21$ For example, global indices such as PSD are spatially insensitive - that is, they cannot distinguish between spatially correlated and uncorrelated (random) damage. In terms of localised field loss, deviation maps and the glaucoma hemifield test $(\mathrm{GHT})^{22}$ appear more intuitive and clinically valid. Importantly, the residuals generated by the image processing technique that form the LSV reported here are amenable to pointwise maps, or plots such as the examples shown in Figure 5. Dark areas indicate locations that are highly at variance with neighbouring points. When Werner and Drance 4 first described the potential utility of threshold variations in predicting frank glaucomatous change the variations were local and not global. The types of plots described here could provide the clinician with an aid to interpreting the spatial configuration of variable locations that may be an early characteristic of glaucomatous field loss.

In summary, image processing techniques can be utilised to describe the threshold behaviour and spatial properties of field data. The LSV extracted by these processes correlates well with conventional parameters. LSV is determined without normals' reference data or replicate threshold measurement, thus saving test time. Moreover, the process could also provide clinically useful spatial maps of the non-uniform deviations and fluctuations within the field. These methods could, after further development and validation, be added to current perimeter software as a statistical bolt on and prove valuable in the detection and management of glaucomatous field loss.

This work was supported by grants from the City Educational Trust, Royal National Institute for the Blind, the Science and Engineering Research Council, the National Retinitis Pigmentosa Foundation, the Wellcome Trust, the Medical Research Council, and the Friends of Moorfields.

1 Flammer J, Drance SM, Fankhauser F, Augustiny L. Differential light threshold in automated static perimetry: factors influencing short-term fluctuation. Arch Ophthalmol 1984; 102: 876-9.

2 Heijl A. A simple routine for demonstrating increased scatter by comparing stored computer fields. Doc Ophthalmol Proc Series 1985; 42: 35-8.

3 Hoskins HD, Magee SD, Drake MV, Kidd MN. A system for the analysis of visual fields using the Humphrey visual field analyzer. Doc Ophthalmol Proc Series 1987; 49: 145-51.

4 Werner EB, Drance SM. Early visual field disturbances in glaucoma. Arch Ophthalmol 1977; 95: 1173-5.

5 Heijl A, Lindgren G, Olsson J. A package for the statistical analysis of visual fields. Doc Ophthalmol Proc Series 1987 49: 153-68.

6 Heijl A, Lindgren G, Olsson J. Normal variability of static perimetric threshold values across the central visual field. Arch Ophthalmol 1987; 105: 1544-9.

7 Iwase A, Kitazawa Y, Ohno Y. On age-related norms of the visual field. Fpn f Ophthalmol 1988; 32: 429-37.

8 Vivell PM, Lachenmayr BJ, Ostermaier N. Normal visual field data for the Humphrey field analyzer. ARVO abstracts. Invest Ophthalmol Vis Sci 1993; 34 (suppl): 2748 .

9 Searle AET, Wild JM, Shaw DE, O'Neil EC. Time related variation in normal automated static perimetry. Ophthalmology 1991; 98: 701-7.

10 Wild JM, Searle AET, Dengler-Harles M, O'Neil EC. Long-term follow-up of baseline learning and fatigue effects in the automated perimetry of glaucoma and ocula hypertensive patients. Acta Ophthalmol 1991; 69: 210-6.

11 Heijl A, Drance SM. Changes in differential threshold in patients with glaucoma during prolonged perimetry. Br ₹ Ophthalmol 1983; 67: 512-6.

12 Heijl A, Lindgren A, Lindgren G. Inter-point correlations of deviations of threshold values in normal and glaucomatous visual fields. In: Heijl A, ed. Perimetry update matous visual fields. In: Heijl A, ed. Perimetry update

13 Lachenmayr B, Kiermeir U, Kojetinsky S. Neighbouring points of a normal visual field are not statistically inpoints of a normal visual field are not statistically in 1994; 35 (suppl): 313.

14 Asman P, Heijl A, Olsson J, Rootzen H. Spatial analyses of glaucomatous visual fields; a comparison with traditional visual field indices. Acta Ophthalmol 1992; 70: 679-86.

15 Fitzke FW, Crabb DP, McNaught AI, Edgar DF, Hitchings RA. Image processing of computerised visual field data. $\mathrm{Br} \mathcal{f}$ Ophthalmol 1995; 79; 207-12. 
16 Bebie H, Frankhauser F, Spahr J. Static perimetry: accuracy and fluctuations. Acta Ophthalmol 1976; 54: 339-48.

17 Flanagan JG, Wild JM, Trope GE. The visual field indices in primary open-angle glaucoma. Invest Ophthalmol Vis Sci 1993; 34: 2266-74.

18 Chauhan BC, LeBlanc RP, Drance SM, Wijsman K, Cruz AM. Effect of the number of threshold determinations on short-term fluctuation in automated perimetry. Ophthalmology 1991; 98: 1420-4.

19 Schulzer M, Mills RP, Hopp RH, Lau W, Drance SM. Estimation of the short-term fluctuation from a single determination of the visual field. Invest Ophthalmol Vis Sci 1990; 31: 730-5.

20 Hitchings RA. Psychophysical testing in glaucoma. $\mathrm{Br} f$ Ophthalmol 1993; 77: 471-2.

21 Johnson CA. Modern developments in clinical perimetry. Johnson CA. Modern developments

22 Heijl A, Lindgren G, Lindgren A, Olsson J, Asman P, Myers $S$, et al. Extended empirical statistical package for evaluation of single and multiple fields in glaucoma. In Mills RP, Heijl A, eds. Perimetry update 1990/91. Amsterdam: Kugler and Ghedini, 1991: 303-15. 